\title{
Effective Power Allocation Using for Transmit Source In MIMO Relay Communication
}

\author{
Hoai Trung Tran
}

\begin{abstract}
The Multiple Input Multiple Output (MIMO) systems using relays are of interest for high-speed radio communication systems. Currently, most of the articles are interested in the model of three nodes with purposes such as increasing the channel capacities (mutual information) or reducing the minimum mean square of error. This paper extends to more than one relay and is concerned with the maximum channel capacity. It is assumed that the channel matrices between source and relay as well as relay and receiver are random matrices; the relay precoders are also assumed to be random and known at the receiver. The article proposes that the Lagrange multiplier finding algorithm using the Newton Raphson optimization method is more straightforward than the traditional finding algorithm using the first and second derivatives but still gives a higher channel capacity.
\end{abstract}

Index Terms - Channel capacity, Lagrangian operator, MIMO, relay.

\section{INTRODUCTION}

Relay-based transmission leads to the advantages of high system reliability, large throughput, and wide coverage in radio communications [1]. In a two-way amplify-and-forward (AF) relay, both terminals know their transmitting signal, and they can ignore self-noise from the received signal before detecting the requested signal if channel state information (CSI) available [1]. When AF is used, the relay amplifies the received signals to overcome the power loss and subsequently forwards them to the destination. In general, AF needs less signal processing at the relay and, thus, is less complex [2], [3].

Combining MIMO with relay-based transmission can better improve system quality due to the advantages of spatial diversity and diversity gain. The MIMO transmission technology is well known to improve spectral efficiency by transmitting multiple data streams across multiple antennas. Multiple antennas could be installed at the source (S), the relay (R), and the destination (D) to form a MIMO relay network [2], [4]. Amongst the various existing relay-based communication strategies (e.g., amplify-and-forward (AF), decode-and-forward, compress-and-forward), AF remains one of the most popular strategies given its simplicity and practicality for enabling multi-input multi-output (MIMO) cooperative communication [4]-[5].

Articles are focused on designing precoders at both source and relay [3], [6], [7]. They also only give a simple model of 3 nodes [4], [5], [7], adding a direct link and no direct link [3].

Submitted on June 09, 2021.

Published on June 30, 2021

Hoai Trung Tran, University of Transport and Communications, Vietnam.

(corresponding e-mail: trungth ${ }^{@}$ utc.edu.vn)
They are also interested in the simultaneous design of the precoder at the transmitter and the combined relay. In addition, figuring out how to allocate and update power is introduced through [4] using the Lagrange method, which follows the water-filling principle. [6] also added a complex algorithm that guarantees to find the multipliers of the Lagrange method. Here, finding this parameters value is sometimes difficult when the algorithm goes through many loops to find. The article [2] presents a precoder design model for relay only, considering channel state information.

Among the above works, there are few works related to the use of the Lagrange algorithm, such as [2], [4]-[7]. [2] is considered to find the optimal power distribution for the relay. [4] is used to find the optimal power distribution for both transmitter and relay to convert the relay channel MIMO into a multi-channel relay single input single output. However, in [3], [4], to ensure the optimal function property with convexity, it is necessary to keep the power distribution coefficient of the relay or generator constant. The article [7] is more general when giving different optimal cases: only relay precoder or find source precoder when relay precoder is known or vice versa. [6], then study the following case of [7] when finding the optimal source precoder first, then finding the optimal relay precoder.

To summarize, some articles talk about how if joint source/relay precoders make the optimized function convex impossible, it makes it challenging to find the optimization for global [4], [6], [7]. The solution should be separated into the optimizations for the source and relay separately, then the optimized functions for each component are convex [4], [6], [7]. Some articles mentioned Lagrange or KKT conditions for optimal source or relay [4]-[7]. However, with the waterfilling algorithm, as shown in [6], it is not very easy to give the optimal eigenvectors and the optimal water-filling algorithm because many conditions need to be satisfied. One method that does not require too many constraints is the Newton - Raphson method, which is described to find the results of the optimal eigenvectors quickly.

\section{ChANNEl Model}

Suppose we have a channel model with a transmitter and a receiver, plus relays in between them. Source, each of the relays, and receiver are all equipped with multiple antennas to transmit and receive data. 


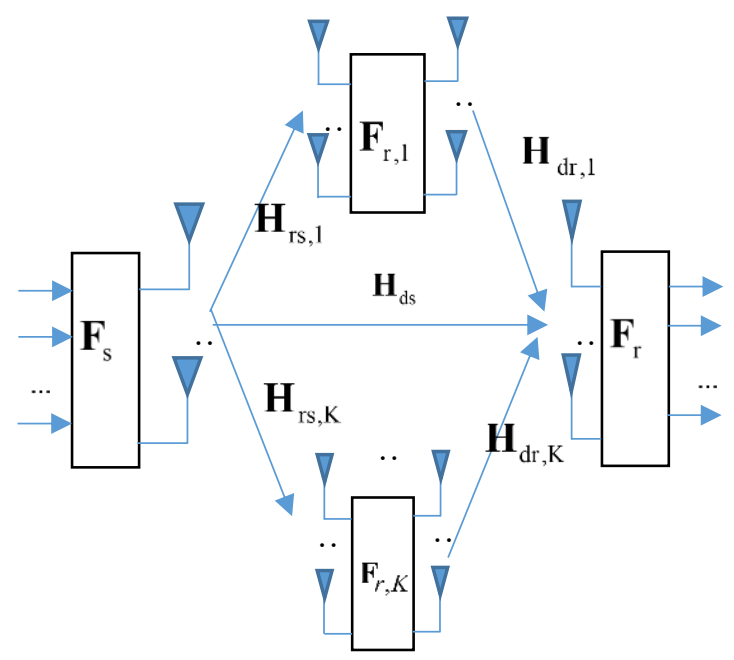

Fig. 1. Channel model consisting of $K$ relays.

Signals received from direct path:

$\mathbf{y}_{d, 1}=\sqrt{\rho_{d s}} \mathbf{H}_{d s} \mathbf{F}_{s} \mathbf{d}+\mathbf{n}_{d s}$

Here $\rho_{\mathrm{ds}}$ is the power distribution factor for the direct link, d is the data vector to be transmitted, $\mathbf{F}_{s}$ is the source precoder, $\mathbf{H}_{d s}$ is the channel matrix between the source and the receiver, and $\mathbf{n}_{d s}$ is the noise acting on the transmission from the source to the receiver.

The signal is received through the relay $k=1: K$ :

$$
\mathbf{y}_{d, 2}=\sqrt{\rho_{d s}} \mathbf{H}_{d r} \mathbf{F}_{r} \mathbf{y}_{r}+\mathbf{n}_{d r}
$$

$$
\text { Here } \mathbf{F}_{r}=\left[\mathbf{F}_{r, 1}^{T}, \mathbf{F}_{r, 2}^{T}, \ldots, \mathbf{F}_{r, K}^{T}\right]^{T} \text {. }
$$

$\mathbf{y}_{\mathrm{r}}$ is the receive vector matrix of $\mathrm{K}$ relays, denoted by the data vector $\mathbf{y}_{r, k}$ received at the $\mathrm{k}$ th relay (the calculation is the same as for the direct transmission line but only $\mathbf{H}_{r s, k}$, $\mathbf{n}_{r s, k}$, is the channel matrix, the noise between the source and the $\mathrm{k}$ th relay):

$$
\mathbf{y}_{r}=\left[\mathbf{y}_{r, 1}^{T}, \ldots, \mathbf{y}_{r, K}^{T}\right]^{T}, \mathbf{y}_{\mathrm{r}, \mathrm{k}}=\sqrt{\rho_{\mathrm{rs}}} \mathbf{H}_{\mathrm{rs}, \mathrm{k}} \mathbf{F}_{\mathrm{s}} \mathbf{d}+\mathbf{n}_{\mathrm{rs}, \mathrm{k}}
$$

Similarly, $\mathbf{H}_{d r}$ is the matrix of the channel matrices between the relay and the receiver: $\mathbf{H}_{d r, k}, k=1: K$ :

$$
\mathbf{H}_{d r}=\left[\mathbf{H}_{d r, 1}, \mathbf{H}_{d r, 2}, \ldots, \mathbf{H}_{d r, K}\right]
$$

The total signal at the receiver:

$$
\mathbf{y}=\left[\mathbf{y}_{d, 1}^{T}, \mathbf{y}_{d, 2}^{T}\right]^{T}=\mathbf{H}_{e f f} \mathbf{d}+\mathbf{n}_{e f f}
$$

where $\mathbf{H}_{e f f}=\left[\begin{array}{c}\sqrt{\rho_{d s}} \mathbf{H}_{d s} \mathbf{F}_{s} \\ \sqrt{\rho_{r s} \rho_{d r}} \mathbf{H}_{d r} \mathbf{F}_{r, n} \mathbf{H}_{r s} \mathbf{F}_{s}\end{array}\right]$ and $\mathbf{n}_{e f f}=\left[\begin{array}{c}\mathbf{n}_{d s} \\ \sqrt{\rho_{d r}} \mathbf{H}_{d r} \mathbf{F}_{r, n} \mathbf{n}_{r s}+\mathbf{n}_{d r}\end{array}\right]$,

and $\mathbf{H}_{r s}=\left[\mathbf{H}_{r s, 1}^{T}, \mathbf{H}_{r s, 2}^{T}, \ldots, \mathbf{H}_{r s, K}^{T}\right]^{T}$,

$\mathbf{n}_{r s}=\left[\mathbf{n}_{r s, 1}^{T}, \mathbf{n}_{r s, 2}^{T}, \ldots, \mathbf{n}_{r s, K}^{T}\right]^{T}$

From there, [6] estimates the channel capacity L as follows:

$$
\begin{aligned}
& \mathbf{P 1}: \max L=\underset{\mathbf{F}_{s}}{\log \operatorname{det}}\left(\mathbf{I}_{N_{s}}+\mathbf{F}_{s}^{H} \mathbf{M}_{\mathbf{F}_{r}} \mathbf{F}_{s}\right) \\
& \text { s.t. } \operatorname{tr}\left(\mathbf{F}_{s}^{H} \mathbf{F}_{s}\right)=1, \operatorname{tr}\left(\mathbf{F}_{s}^{H} \mathbf{B} \mathbf{F}_{s}\right)=1
\end{aligned}
$$

where $\mathbf{M}_{\mathbf{F}_{r}}$ is calculated as follows:

$$
\begin{aligned}
\mathbf{M}_{\mathbf{F}_{r}} & =\mathbf{G}_{d s}+\mathbf{G}_{r s}-\rho_{r s}\left[\overline{\mathbf{H}}_{r s}^{H} \boldsymbol{\Theta}_{F_{r}} \overline{\mathbf{H}}_{r s}+\frac{\operatorname{tr}\left(\boldsymbol{\Theta}_{F_{r}} \boldsymbol{\Sigma}_{r}^{H}\right)}{1+K_{r s}} \mathbf{\Psi}_{s}^{*}\right] \\
G_{a b} & =\rho_{a b} E\left[\mathbf{H}_{a b}^{H} \mathbf{H}_{a b}\right]=\rho_{a b} E\left[\overline{\mathbf{H}}_{a b}^{H} \overline{\mathbf{H}}_{a b}+\frac{\operatorname{tr}\left(\Sigma_{a}^{H}\right)}{1+K a b} \mathbf{\Psi}_{b}^{*}\right] \\
\boldsymbol{\Theta}_{F_{r}} & =\left(\mathbf{I}_{N_{r}}+\mathbf{F}_{r}^{H} \mathbf{G}_{d r} \mathbf{F}_{r}\right)^{-1}
\end{aligned}
$$

and

$$
\mathbf{B}=\frac{\rho_{r s}}{1-\operatorname{tr}\left(\mathbf{F}_{r}^{H} \mathbf{F}_{r}\right)}\left[\stackrel{-{ }^{H}}{\mathbf{H}_{r s}} \mathbf{F}_{r}^{H} \mathbf{F}_{r} \overline{\mathbf{H}}_{r s}+\frac{\operatorname{tr}\left(\mathbf{F}_{r}^{H} \mathbf{F}_{r} \boldsymbol{\Sigma}_{r}\right)}{1+K_{r s}} \mathbf{\Psi}_{s}^{T}\right]
$$

\section{CURRENT Algorithm FOR THE SOURCE}

With [6], we can find the optimum eigenvectors and eigenvalues for the source. Optimum source beam eigenvectors are the transpose conjugate of the eigenvectors of $\mathbf{M}_{\mathbf{F}_{r}}=\mathbf{U}_{M} \boldsymbol{\Lambda}_{M} \mathbf{U}_{M}^{H}$.

The eigenvalues of the corresponding eigenvectors of the relay are $\boldsymbol{\Lambda}_{M}=\operatorname{diag}\left\{\sigma_{M, 1}, . ., \sigma_{M, N_{s}}\right\}$ so that $\left\{\sigma_{M, i}\right\}$ sorted in descending order.

$\mathbf{P} 2: \min _{x} J=-\sum_{i=1}^{N_{s}} \log \left(1+\sigma_{M, i} x_{i}\right)$

s.t. $\sum_{i=1}^{N_{s}} x(i)=1, \sum_{i=1}^{N_{s}} x_{i} b_{i}=1 ; x_{i} \geq 0, i=1, \ldots, N_{s}$

where $\mathbf{x}=\left[x_{1}, \ldots, x_{N_{s}}\right]=\left[\lambda_{s, 1}^{2}, \ldots ., \lambda_{s, N_{2}}^{2}\right]$ is the vector of the square of the power control factor of $\boldsymbol{\Lambda}_{s}$ and $b_{i}$ is the $\mathrm{i}$ th element of $\mathbf{U}_{M}^{H} \mathbf{B} \mathbf{U}_{M}$.

The problem is divided into the following cases based on the Lagrange operator for (7): 


$$
\begin{aligned}
& L\left(v_{1}\right)=-\sum_{i \in I_{0}} \log \frac{a_{2} \sigma_{M, i}}{\left(a_{2}-a_{1} b_{i}\right) v_{1}+\left|I_{o}\right| b_{i}} \\
& L^{\prime}\left(v_{1}\right)=\sum_{i \in I_{0}} \frac{a_{2}-a_{1} b_{i}}{\left(a_{2}-a_{1} b_{i}\right) v_{1}+\left|I_{o}\right| b_{i}} \\
& L^{\prime \prime}\left(v_{1}\right)=-\sum_{i \in I_{0}} \frac{\left(a_{2}-a_{1} b_{i}\right)^{2}}{\left[\left(a_{2}-a_{1} b_{i}\right) v_{1}+\left|I_{o}\right| b_{i}\right]^{2}}
\end{aligned}
$$

with $\left|\mathrm{I}_{0}\right|=\mathrm{N}_{\mathrm{s}}$

$$
a_{1}=1+\sum_{i \in \mathbf{I}_{0}} \sigma_{M, i}^{-1}>0, a_{2}=1+\sum_{i \in \mathbf{I}_{0}} b_{i} \sigma_{M, i}^{-1}>0
$$

1. $v_{l b}<v_{u b}$ then:

when $L^{\prime}\left(v_{l b}\right) \leq 0$, we have $\mathrm{v}_{1}^{\text {opt }}=\mathrm{v}_{\mathrm{lb}}$. If $\mathrm{v}_{1}^{\mathrm{opt}}=\mathrm{v}_{\mathrm{lb}}=\mathrm{v}_{\mathrm{lb}}^{\mathrm{j}}$ then $\mathbf{I}_{0}=\mathbf{I}_{0} / j$. Continue to calculate for new $\mathbf{I}_{0}$.

when $L^{\prime}\left(v_{u b}\right) \geq 0$, we have $\mathrm{v}_{1}^{\mathrm{opt}}=\mathrm{v}_{\mathrm{ub}}$. If $v_{1}^{\text {opt }}=v_{u b}=v_{u b}^{j}$ then $\mathbf{I}_{0}=\mathbf{I}_{0} / j$. Continue to calculate for new $\mathbf{I}_{0}$.

when $L^{\prime}\left(v_{l b}\right)>0$ and $L^{\prime}\left(v_{u b}\right)<0$, then $\mathrm{v}_{1}^{\text {opt }}$ will make $L^{\prime}\left(v_{1}^{\text {opt }}\right)=0$.

2. $v_{l b}>v_{u b}$, then $\mathbf{I}_{0}$ is modified.

3. $v_{l b}=v_{u b}$, then $v_{1}^{o p t}=v_{l b}=v_{u b}$.

4. Find $x_{i}=a_{2}\left[\left(a_{2}-a_{1} b_{i}\right) v_{1}^{o p t}+\left|I_{o}\right| b_{i}\right]^{-1}-\sigma_{M, i}^{-1}$

Note:

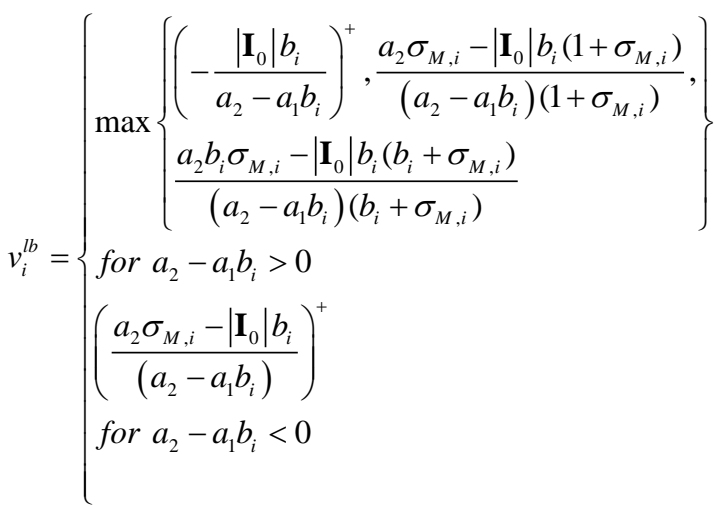

$$
\begin{aligned}
& v_{i}^{u b}=\left\{\begin{array}{l}
\left(\frac{a_{2} \sigma_{M, i}-\left|\mathbf{I}_{0}\right| b_{i}}{\left(a_{2}-a_{1} b_{i}\right)}\right)^{-} \\
\text {for } a_{2}-a_{1} b_{i}>0 \\
\min \left\{\begin{array}{l}
\left(-\frac{\left|\mathbf{I}_{0}\right| b_{i}}{a_{2}-a_{1} b_{i}}\right)^{+}, \frac{a_{2} \sigma_{M, i}-\left|\mathbf{I}_{0}\right| b_{i}\left(1+\sigma_{M, i}\right)}{\left(a_{2}-a_{1} b_{i}\right)\left(1+\sigma_{M, i}\right)} \\
\frac{a_{2} b_{i} \sigma_{M, i}-\left|\mathbf{I}_{0}\right| b_{i}\left(b_{i}+\sigma_{M, i}\right)}{\left(a_{2}-a_{1} b_{i}\right)\left(b_{i}+\sigma_{M, i}\right)}
\end{array}\right. \\
\text { for } a_{2}-a_{1} b_{i}<0
\end{array}\right\} \\
& v_{l b}=\max _{i \in \mathbf{I}_{0}}\left\{v_{i}^{l b}\right\} \text { and } v_{u b}=\min _{i \in \mathbf{I}_{0}}\left\{v_{i}^{u b}\right\}
\end{aligned}
$$

\section{PROPOSED Method}

According to [8], page 66 mentions that the NewtonRaphson method is considered a Newton quasi method, has the advantage of fast convergence, and is suitable for fast fading environments.

The steps for Newton- Raphson are applied as below:

1. The initial decision value $v_{1}(1)=0$

$$
\begin{aligned}
& L^{\prime}\left(v_{1}(1)\right)=\sum_{i \in I_{0}} \frac{a_{2}-a_{1} b_{i}}{\left(a_{2}-a_{1} b_{i}\right) v_{1}(1)+\left|I_{o}\right| b_{i}} \\
& L^{\prime \prime}\left(v_{1}(1)\right)=-\sum_{i \in I_{0}} \frac{\left(a_{2}-a_{1} b_{i}\right)^{2}}{\left[\left(a_{2}-a_{1} b_{i}\right) v_{1}(1)+\left|I_{o}\right| b_{i}\right]^{2}}
\end{aligned}
$$

2. Update value:

$v_{1}(i+1)=v_{1}(i)-\frac{L^{\prime}\left(v_{1}(i)\right)}{L^{\prime \prime}\left(v_{1}(i)\right)}$

3. Find $v_{1}^{o p t}=v_{1}(i)$ so that $\left|v_{1}(i)-v_{1}(i+1)\right| \leq \varepsilon$ or

$$
\left|L^{\prime}\left(v_{1}(i+1)\right)\right| \leq \varepsilon
$$

Find

$$
x_{i}=a_{2}\left[\left(a_{2}-a_{1} b_{i}\right) v_{1}^{o p t}+\left|I_{o}\right| b_{i}\right]^{-1}-\sigma_{M, i}^{-1}
$$

\section{Simulation}

We assume that the model consists of 2 relays, each with four transmit and four receive antennas. Likewise, the transmitter and receiver have an antenna count of 4 . We assume channel matrices with random fading coefficients like $\mathbf{H}_{r s, k} \mathbf{H}_{d r, k}, k=1: 2$, as even the two relay precoders $\mathbf{F}_{r, k}, k=1: 2$ are considered arbitrary. Based on (4), we can find the source beam eigenvectors of the matrix by matrix. Specifically, we consider the case of using algorithm [6] to increase the capacity; then we are assuming that the beam eigenvectors (number of 4) $\mathbf{F}_{s}$ are equal to the conjugate transpose of the correlated matrix $\mathbf{M}_{\mathbf{F}_{r}}$ 's eigenvectors, we have two cases: Transmitted power values $\mathrm{x}_{\mathrm{i}}, \mathrm{i}=1: 4$, are taken in absolute value (marked "o," solid- Case A), and when negative values of $x_{i}, i=1: 4$ return to 0 are the second case (marked "o", dashed- Case B).

Meanwhile, with the proposed Newton- Raphson algorithm applied to find $x_{i}, i=1: 4$, we have a line marked "x" dashed (Case B) which characterizes values $x_{i}, i=1: 4$ as 0 when they are negative, the remaining line "x," solid (Case A) is the case of taking the absolute value of $x_{i}, i=1: 4$.

Similarly, the solid or dashed line, marked "v" is characteristic of the algorithm [6]; however, the generator has random eigenvectors, which are 0 when it is negative (Case B) or absolute value (Case A). Solid or dashed line, marked with "+" is typical in the case of Newton- Raphson's proposed algorithm; however, the generator has random eigenvectors 
(at the moment the receiver only send to the source eigenvalue, corresponding to the gains of expected channels), which is 0 when it is negative (Case B) or its absolute value (Case A).

When we randomize the channel matrices like $\mathbf{H}_{r s, k} \mathbf{H}_{d r, k}, k=1: 2$, and also the two relay precoders for 5 times, we get the diagram as Fig. 2. Here, in all 5 cases, the capacity of the proposed algorithm (Newton- Raphson) is the most significant and equal, marked " $\mathrm{x}$ " (in the case of taking the absolute value - Case A and taking the value of 0 when negative power-Case B). In the case of the first, fourth and fifth randomization, the capacity of the proposed algorithm has the most significant value when SNR has a high value. The capacity of other cases, including algorithm [6], marked "o" when the source eigenvectors are known (Case A and Case B) or marked " $v$ " randomly taken at the source (Case A and Case B), proposed algorithm marked "+" the source eigenvectors known randomly (Case $\mathrm{A}$ and Case B), is confused to be better among algorithms. This is also because when randomly taking the source eigenvectors, it is possible that in some cases, they coincide with the ideal source eigenvectors, thus giving a high capacity.

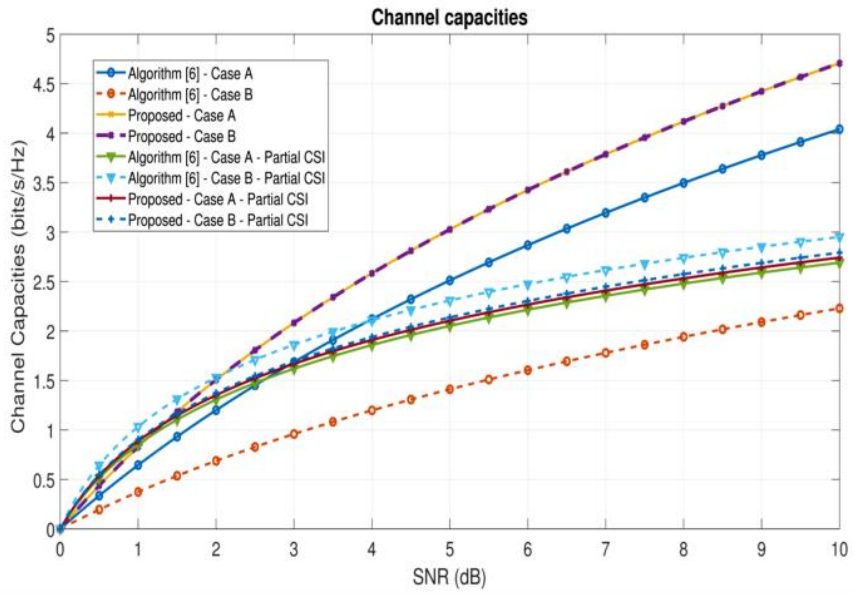

(1)

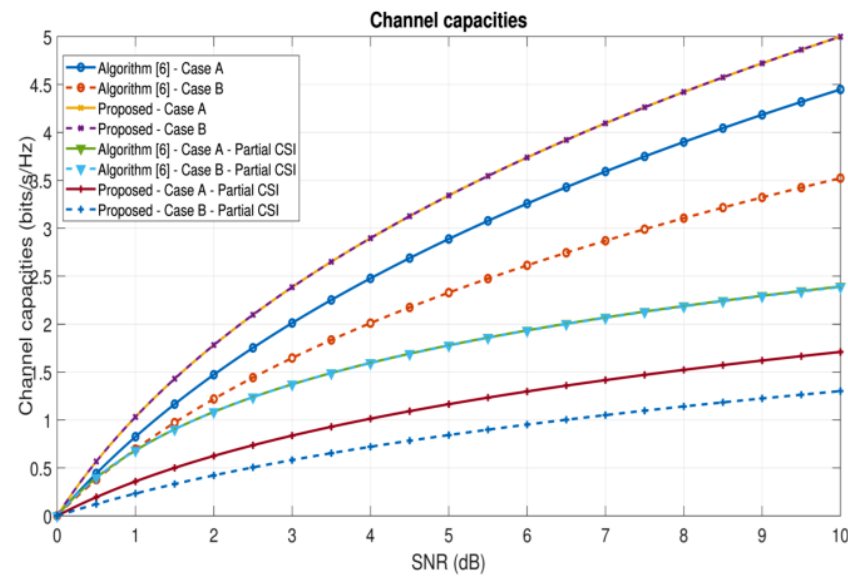

(3)

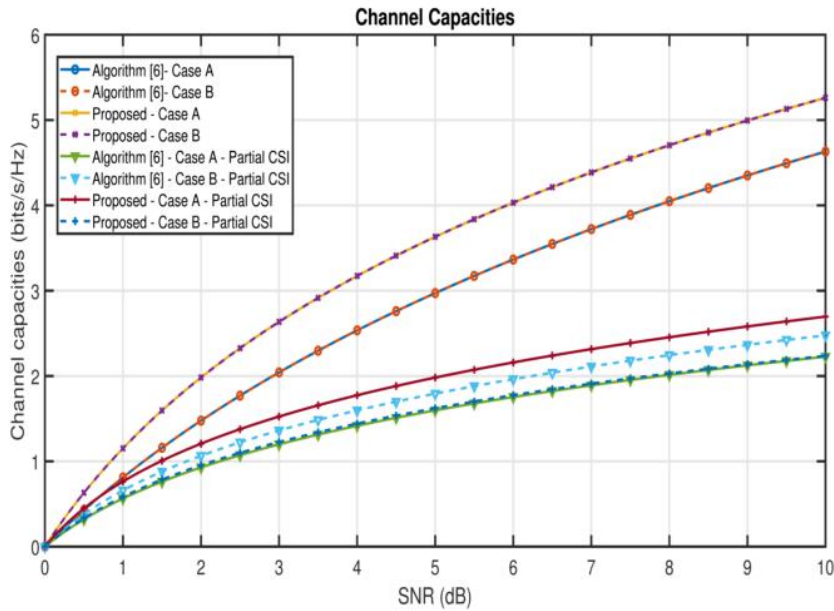

(2)

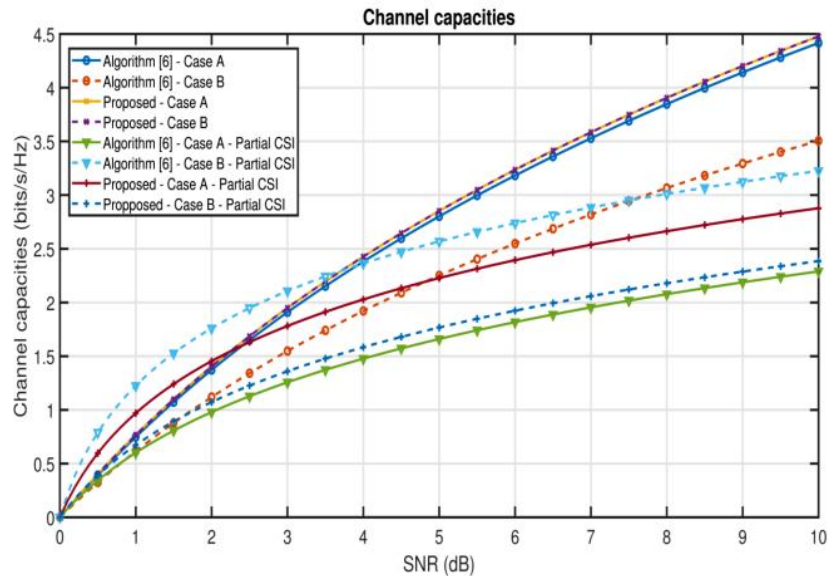

(4)

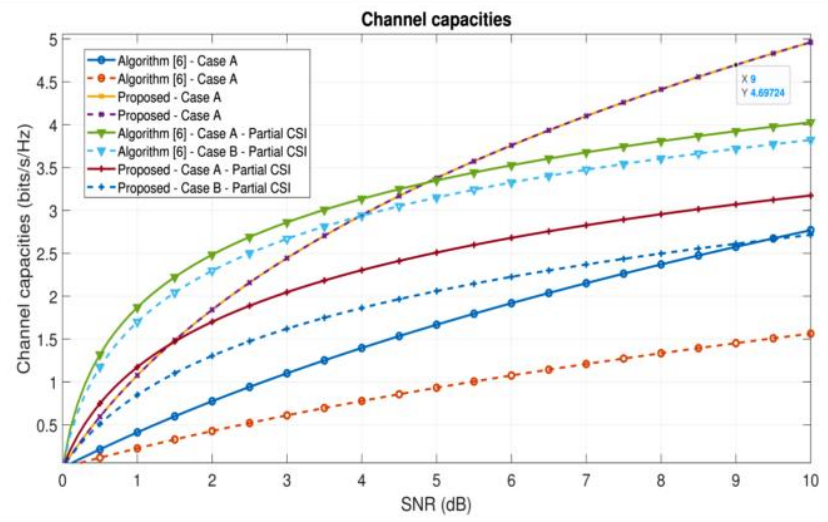

(5)

Fig. 2. Channel capacities for proposed algorithm and algorithm [6]. 


\section{CONCLUSION}

MIMO systems using relays are receiving much attention due to their ability to increase capacity and reduce transmission errors. However, complexity increases as we need to find the optimal source and relay precoders to get the highest capacity. The paper focuses on the simpler algorithm to find the optimum power allocation at the source precoder, assuming that the optimal source beam eigenvectors are perfectly known. This algorithm is more uncomplicated than the algorithm [6], while in many testing attempts, it gives higher capacity than the algorithm [6].

\section{REFERENCES}

[1] Chin-Liang Wang, Jyun-Yu Chen, Jhih-Jhong Jheng, “A precoder design for two-way amplify-and-forward MIMO relay systems with linear receivers", IEEE 80th Vehicular Technology Conference (VTC2014-Fall), Jan. 2014.

[2] Ronghong Mo, Yong Huat Chew, and Chau Yuen, Senior, "Information rate and relay precoder design for amplify-and-forward MIMO relay networks with imperfect channel state information", IEEE Transactions On Vehicular Technology, Vol. 61, no. 9, Nov. 2012.

[3] Lisi Jiang, Hamid Jafarkhani, "MmWave Amplify-and-Forward MIMO Relay Networks with Hybrid Precoding/Combining Design", IEEE Transactions on Wireless Communications, Vol. 19, issue. 2, pp 1333- 1346, Feb. 2020.
[4] Yang Zhang, Jiandong Li, Lihua Pang and Zhi Ding, "Join optimization of source and relay precoding in non-regenerative MIMO relay systems", Wirel. Commun. Mob. Comput., 2012

[5] Fabien Héliot and Rahim Tafazolli, "Optimal Energy-Efficient Source and Relay Precoder Design for Cooperative MIMO-AF Systems", IEEE Transactions on Signal Processing, Vol. 66, issue. 3, pp. 573588, Feb. 2018.

[6] Rongbin Zhang, Shu-Hung Leung, Zhen Luo, and Hong Wan "Precoding Design for Correlated MIMO-AF Relay Networks With Statistical Channel State Information", IEEE Transactions on Signal Processing, Vol. 66, No. 22, Nov 2018

[7] Ronghong Mo, and Yong Huat Chew, "Precoder Design for NonRegenerative MIMO Relay Systems", IEEE Transactions on Wireless Communications, Vol. 8, no. 10, pp. 5041-5049, Oct 2009.

[8] Urmila Diwekar, Introduction To Applied Optimization, 2nd ed. Springer Science+Business Media, LLC, 2008.S. Chen, B. Mulgrew, and P. M. Grant, "A clustering technique for digital communications channel equalization using radial basis function networks," IEEE Trans. on Neural Networks, vol. 4, pp. 570-578, July 1993.

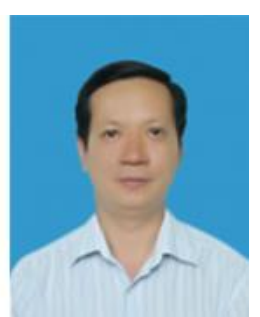

Hoai Trung Tran was born in 1976. He got Bachelor degree in University of Transport and Communications (UTC) in 1997 and hold the post of lecturer at the University. He then got a Master degree from Hanoi University of Science and Technology (HUST) in 2000. In the period 2003 to 2008 , he had concentrated on researching in the field of Telecommunication engineering and got his $\mathrm{PhD}$ at University of Technology, Sydney (UTS) in Australia. He is currently lecturer at the UTC. His main research interests are digital signal processing (DSP), applied information theory, radio propagation, MIMO antenna techniques and advanced wireless transceiver design. 